\title{
Qualidade de vida, estresse e desempenho ocupacional de mães cuidadoras de crianças e adolescentes autistas*
}

\section{Quality of life, stress and occupational performance of caregiver mothers of autistic children and adolescents}

\author{
Ingrid Ioost Estanieski ${ }^{1}$, Nicole Ruas Guarany ${ }^{2}$
}

http://dx.doi.org/10.11606/issn.2238-6149.v26i2p194-200

Estanieski II, Guarany NR. Qualidade de vida, estresse e desempenho ocupacional de mães cuidadoras de crianças e adolescentes autistas. Rev Ter Ocup Univ São Paulo. 2015 maio-ago.;26(2):194-200.

RESUMO: O objetivo deste estudo é avaliar a qualidade de vida, estresse e o desempenho ocupacional de mães cuidadores de crianças e adolescentes com diagnóstico de autismo. Para a pesquisa, foram utilizados quatro instrumentos: WHOQOL-Bref, a Escala de Estresse Percebido, a Medida Canadense de Desempenho Ocupacional e um questionário criado pela pesquisadora contendo dados socioeconômicos e sobre a criança/adolescente. Os dados do estudo sugerem que existe correlação entre as variáveis e que as mães cuidadoras possuem menor qualidade de vida, altos níveis de estresse e desempenho ocupacional baixo.

DESCRITORES: Qualidade de vida; Estresse psicológico; Análise e desempenho de tarefas; Transtorno autístico; Cuidadores/ psicologia; Mães/psicologia; Autocuidado/psicologia; Atividades humanas/psicologia; Terapia ocupacional.
Estanieski II, Guarany NR. Quality of life, stress and occupational performance of caregiver mothers of autistic children and adolescents. Rev Ter Ocup Univ São Paulo. 2015 May-Aug.;26(2):194-200.

ABSTRACT: The objective of this study is to evaluate the quality of life, stress, and occupational performance of caregiver mothers of children and adolescents diagnosed with autism. For the research, four instruments were used: WHOQOL-Bref, the Perceived Stress Scale, the Canadian Occupational Performance Measure and a questionnaire created by the researcher containing socioeconomic data and information about the child/adolescent. The study data suggest that there is a correlation between the variables and that caregiver mothers have a lower quality of life, high levels of stress, and low occupational performance.

KEYWORDS: Quality of life; Stress, psychological; Task performance and analysis; Autistic disorder; Caregivers/ psychology; Mothers/psychology; Self care/psychology; Human activities/psychology; Occupational therapy.

\footnotetext{
* Trabalho apresentado à banca examinadora como requisito parcial à obtenção do título de Bacharel em Terapia Ocupacional pela Universidade Federal de Pelotas.

1. Bacharel em Terapia Ocupacional, Universidade Federal de Pelotas.

2. Professora Assistente I do Departamento de Terapia Ocupacional da Universidade Federal de Pelotas.

Endereço para correspondência: Nicole Ruas Guarany. Universidade Federal de Pelotas - Curso Superior de Bacharelado em Terapia Ocupacional. Avenida Duque de Caxias, n² 250, Fragata, Pelotas, RS, Brasil. CEP: 96030-000. E-mail: to.ufpel@gmail.com.
} 


\section{INTRODUÇÃO}

A utismo é um transtorno global de desenvolvimento que tem como característica inaptidão para interagir socialmente e alterações comportamentais ${ }^{1}$. O desenvolvimento neuropsicomotor manifesta-se inadequado antes dos 3 anos de idade, atrasando algumas etapas do desenvolvimento. Há prejuízo na interação social, na comunicação e padrões estereotipados de comportamentos repetitivos e interesses ${ }^{2}$, que, somados, acarretam em grandes consequências no funcionamento do cotidiano ${ }^{3}$.

Em relação à incidência, ainda não há dados exatos quanto aos países em desenvolvimento. Isso acontece por diversos fatores, entre eles: a falta de dados, ausência de serviços e as barreiras enfrentadas para realizar pesquisas ${ }^{4}$. Especialmente no Brasil a falta de instrumentos validados para diagnóstico e inquirição do autismo colabora para a escassez de estudos, tornando-se principalmente um fator demarcador para profissionais e pesquisadores ${ }^{5}$.

Apesar de todos os avanços em busca da etiologia do autismo, ainda não há consenso entre os pesquisadores. Os estudos desenvolvidos até hoje apontam hipóteses sobre alterações nas estruturas cerebrais. Um estudo realizado em 2006 afirma que o autismo é um transtorno genético, com estimativa de herdabilidade de 90\%. Contudo sua etiologia ainda permanece desconhecida ${ }^{6,7}$, portanto a identificação e o diagnóstico são baseados no comportamento e na história do desenvolvimento de cada indivíduo, não possuindo um marcador biológico que o determine ${ }^{8,9}$. Ainda não existe uma cura conhecida para esse transtorno. Entretanto as intervenções precoces e apropriadas resultam em bom prognóstico ${ }^{10}$.

É importante para o processo de diagnóstico a presença da família, visto que o choque previsto é grande e necessita ser minimizado, já que, ao receber a notícia do diagnóstico, toda a família é atingida ${ }^{11}$.

Os comportamentos característicos dos portadores de autismo junto à gravidade do transtorno podem constituir fatores estressores para os demais membros da família ${ }^{12}$. O estresse familiar pode ser um dos aspectos que explica o estereótipo atribuído aos pais de crianças autistas como sendo emocionalmente frios e apresentando grande dificuldade em criar/manter contato afetivo ${ }^{13}$.

A função de cuidar de uma pessoa com necessidades especiais geralmente recai sobre um familiar. Alguns estudos já descreveram as consequências enfrentadas por cuidadores, tais como: responsabilidade pelo cuidado, isolamento social, ansiedade, carga emocional, entre outros $^{14,15}$, e isso leva à diminuição da qualidade de vida.
A qualidade de vida é um conceito que inclui diversos aspectos. Dentre eles, a sensação de bem-estar, felicidade diante do âmbito em que se vive e suas relações sociais, tais como comunidade, escola, trabalho, família ${ }^{16}$. Sendo assim, o indivíduo raramente é o único foco da intervenção ${ }^{17}$, visto que a família também é impactada diante do forte estresse sofrido no cuidar de uma criança com diagnóstico de autismo, o que acaba culminando em consequências no desenvolvimento dessa, no sistema familiar e principalmente na vida da mãe ${ }^{18}$. Esse impacto ocorre porque o envolvimento nas ocupações humanas sustenta o cotidiano, auxiliando na saúde e bem-estar ${ }^{17}$, e possuir uma criança com deficiência mental na família pode ser um fator estressante ou não, acarretando em consequências para todos os membros da família, em pequena ou grande proporção ${ }^{19}$.

Este estudo tem como objetivo avaliar a correlação entre qualidade de vida, estresse e o desempenho ocupacional de mães cuidadoras de crianças e adolescentes com diagnótisco de autismo.

A pesquisa surgiu a partir da observação da necessidade de um atendimento humanizado com suporte às famílias e, especificamente, aos cuidadores de indivíduos autistas. A ideia de desenvolver o estudo se dá a partir do suporte que os cuidadores de pessoas com necessidade especial devem receber, visto que o cotidiano em que esses se inserem acaba tornando-se conturbado devido às tarefas acumuladas.

O trabalho é de suma importância, visto que não foi encontrado na literatura estudo que correlacione qualidade de vida, estresse e desempenho ocupacional, e este poderá trazer maiores esclarecimentos e referencias bibliográficas para acadêmicos e profissionais que se interessem sobre o tema abordado. A instituição escolhida foi o Centro de Autismo Dr. Danilo Rolim de Moura, que oferece atendimento gratuito a crianças e adolescentes com diagnóstico de autismo, sendo uma oportunidade de conhecer melhor a vida desses cuidadores de pessoas com necessidade especial, apresentando dados que sugerem como é a vida das mães cuidadoras.

\section{PROCEDIMENTOS METODOLÓGICOS}

Trata-se de um estudo transversal de caráter quantitativo, caracterizado por amostra de conveniência. Foi realizado no período de maio a junho de 2013, no Centro de Atendimento ao Autista Dr. Danilo Rolim de Moura, em Pelotas, onde as mães foram recrutadas por meio de uma conversa e convidadas a participar. Foram 
excluídas as mães que apresentaram déficit cognitivo e déficit visual.

Para o estudo, utilizou-se quatro instrumentos para avaliar as variáveis: questionário sobre dados pessoais e socioeconômicos construído pelos pesquisadores; a Medida Canadense de Desempenho Ocupacional (COPM) para avaliar o desempenho ocupacional das mães ${ }^{20}$; o WHOQOL Bref para avaliar a qualidade de $v^{2} \mathrm{da}^{21}$; e a Escala de Percepção de Estresse (EPS-10) para avaliar os níveis de estresse existentes ${ }^{22}$. Em relação às pontuações dos instrumentos, os três questionários em seus manuais de aplicação, indicam que, quanto maior a pontuação, melhor será a qualidade de vida, o desempenho ocupacional e a satisfação do sujeito em realização, e maior será o nível de estresse.

Esta pesquisa foi submetida à Plataforma Brasil e autorizada pelo Comitê de Ética e Pesquisa da Universidade Federal de Pelotas por meio do CAAE: 26642314.2.0000.5317.

As variáveis qualitativas foram descritas na forma de frequência simples e as quantitativas, na forma de mediana e intervalo interquartil. Para identificar os níveis de estresse e o desempenho ocupacional das cuidadoras foram utilizados os escores e categorias padronizadas já definidas de cada instrumento. A correlação entre as variáveis foi realizada pelos testes de Spearman, em razão de a amostra não obedecer a uma distribuição normal. $\mathrm{O}$ nível de significância estabelecido foi de $\mathrm{p}<0,05$. Para a interpretação da magnitude das correlações, adotou-se a seguinte classificação dos coeficientes de correlação: coeficientes de correlação $<0,4$ (correlação de fraca magnitude), $\geq 0,4 \mathrm{a}<0,5$ (de moderada magnitude) $\mathrm{e} \geq 0,5$ (de forte magnitude) ${ }^{23}$.

\section{RESULTADOS E DISCUSSÃO}

A amostra foi composta por 32 mães cuidadoras, com mediana de idade 36 anos (IQ: 32-43 anos); a maior parte possui renda menor de mil reais por mês $(\mathrm{n}=16)$, são casadas $(n=19)$, e a maioria das mães afirmava não ter religião $(\mathrm{n}=10)$.

As principais dificuldades reconhecidas por elas quanto ao desempenho ocupacional e relações interpessoais de seus filhos foram a dificuldade de estabelecer interações sociais com outras crianças, pessoas, objetos ou eventos ( $\mathrm{n}=19)$; dificuldade da comunicação verbal e não-verbal $(\mathrm{n}=16)$; e comportamentos repetitivos e compulsivos $(n=9)$.

O autismo apresenta manifestações como disfunções sociais, dificuldade em socializar, disfunções da linguagem, dificuldade em captar e interpretar sinais de comunicação verbais ou não verbais. As disfunções comportamentais apresentam-se em duas fases: a) comportamentos motores estereotipados e repetitivos; e b) comportamentos cognitivos diferenciados tais como rotinas, rituais, compulsões entre outros ${ }^{24,25,26}$.

A análise da correlação entre as variáveis por meio do teste de correlação de Spearman identificou correlação inversa e moderada entre o escore total do instrumento EPS-10 e o Domínio Físico (r=--457), Domínio Psicológico $(\mathrm{r}=-, 559)$, Domínio autoavaliação de qualidade de vida $(\mathrm{r}=-, 363)$ e Domínio Meio-Ambiente $(\mathrm{r}=-, 620)$ do Whoqol-BREF. Outras correlações entre esses instrumentos não foram significativas.

Tais resultados parecem indicar que quanto mais alto o nível de estresse, menor será a qualidade de vida. Estudos afirmam que o estresse familiar afeta principalmente a vida dos pais e das crianças autistas, havendo impacto negativo na qualidade de vida ligada à saúde dos cuidadores, corroborando que quanto maior a sobrecarga, pior a qualidade de vida, relacionando a qualidade diretamente à sobrecarga dos cuidadores, sendo que a falta de dinheiro, o excesso de responsabilidade e a dependência estão entre os principais fatores do desgaste ${ }^{27,28,29}$.

A avaliação da qualidade de vida por meio do WHOQOL-BREF apresentou menor pontuação no Domínio Meio Ambiente (58,97 pontos); domínio que é composto por questões sobre segurança física e proteção, ambiente no lar, ambiente físico, recursos financeiros, participação de lazer, oportunidades para novas informações e habilidades, cuidados da saúde e sociais. Foi seguido pelo Domínio de Relações Pessoais (64,25 pontos), que contém aspectos sobre as relações pessoais, atividade sexual e apoio/suporte social. O Domínio Psicológico $(65,32)$ é constituído de questões sobre os sentimentos positivos, pensar, aprender, memória e concentração, autoestima, aparência e sentimentos negativos. E o Domínio Físico que apresentou a maior pontuação $(71,66)$, é caracterizado por aspectos relativos a dor e desconforto, energia e fadiga, sono e repouso ${ }^{21}$.

Um estudo realizado com cuidadores de crianças com neoplasia cerebral correlacionou os domínios do WHOQOL-BREF mostrando a influência que cada um exerce na vida dos cuidadores. Os resultados indicaram que o Domínio Meio Ambiente foi o que apresentou menor nota pela avaliação dos cuidadores, porém o Domínio Físico é o que mais influencia na qualidade de vida, seguido do Domínio Social ${ }^{31}$. 
Os cuidadores passam a ter uma grande sobrecarga devido ao ato de cuidar, que na maior parte das vezes leva a uma qualidade de vida debilitada ${ }^{15}$. Um estudo realizado sobre a qualidade de vida, utilizando o mesmo instrumento e que avaliou a sobrecarga em cuidadores de pessoas com deficiência intelectual, aponta que os cuidadores, em sua maior parte, apresentaram menores valores no Domínio Meio Ambiente, seguidos de Domínio Físico e Domínio Psicológico ${ }^{27}$, o que sugere a confirmação de nossos resultados quanto ao impacto dos níveis de estresse do cuidado na qualidade de vida das mães.

A Medida Canadense de Desempenho Ocupacional (COPM) apresentou correlação positiva entre o seu módulo de Desempenho Ocupacional e o Domínio Meio-Ambiente do Whoqol-BREF ( $r=, 220)$ e o módulo de Satisfação com o Desempenho Ocupacional e o Domínio Meio-Ambiente do Whoqol-BREF $(\mathrm{r}=, 312)$. Outras correlações não foram significativas.
Este resultado indica que quanto menor o desempenho ocupacional e satisfação na realização das atividades, menor será a qualidade de vida nas mães de sujeitos autistas. As atividades ocupacionais que deveriam ser desempenhadas pelos cuidadores acabam sendo transferidas ou excluídas da rotina, em razão da sobrecarga existente e da responsabilidade que atendem. Atividades de autocuidado e lazer e atividades pessoais acabam perdendo espaço na vida dos cuidadores, o que torna-se um fator estressor por causa da carga acumulada ${ }^{24}$.

As pontuações dos módulos avaliados pela COMP foram baixas, com média de 4,66 no Módulo de Desempeho Ocupacional e 4,62 no Módulo de Satisfação do Desempenho em um total de 10 pontos máximos. As maiores dificuldades de desempenho ocupacional relatadas pelas mães foram: realizar atividades de lazer, realizar atividades de autocuidado - como comparecer a consultas médicas -, cuidar da aparência estética, entre outros, e relaxar no que se refere a ter um tempo de descanso em sua própria companhia (tempo só) (Figura 1).

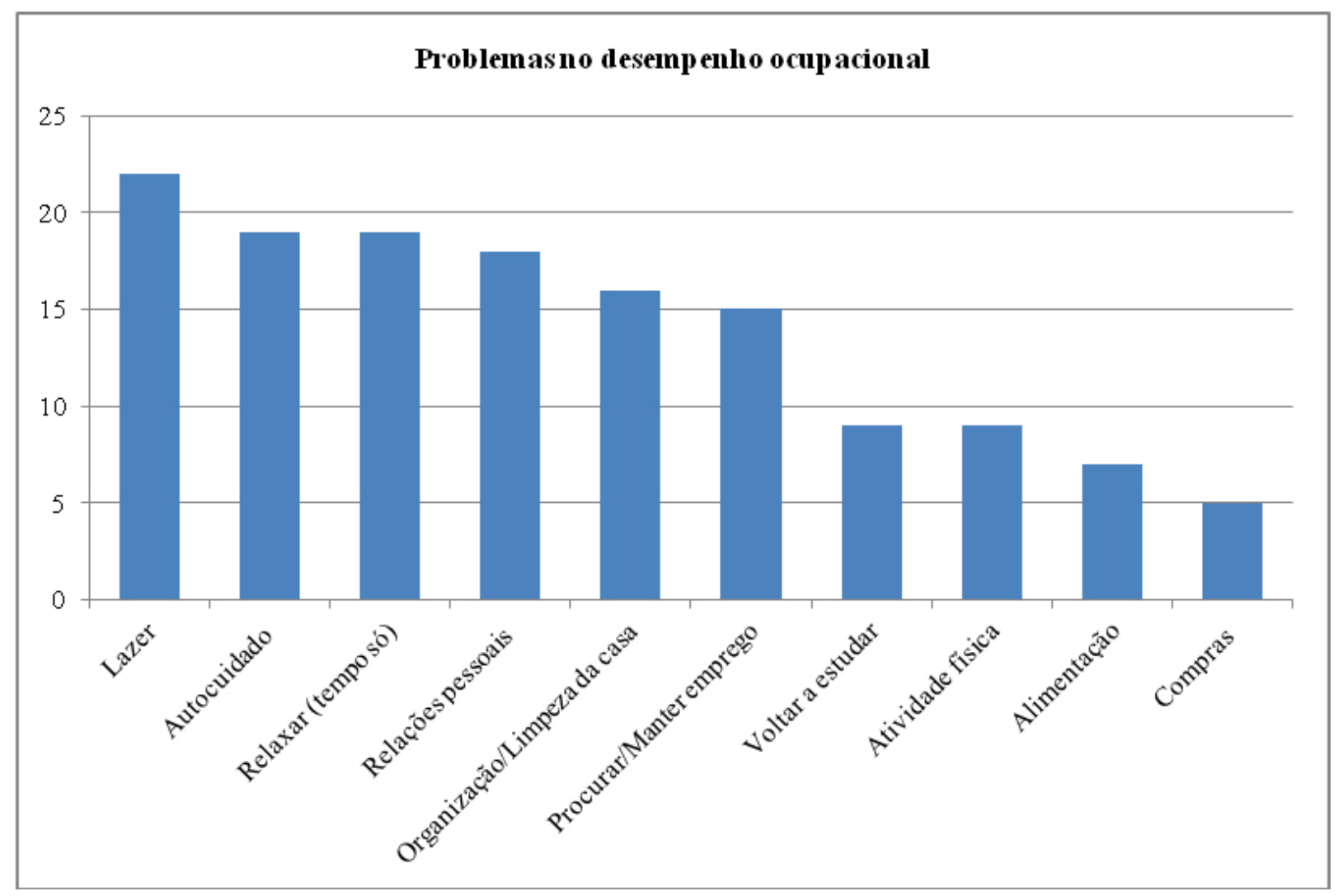

Figura 1 - Problemas no desempenho profissional 
Segundo relato das mães, quase nunca há tempo para cuidar de si, seja para realizar uma atividade de que gosta, seja para desempenhar uma atividade de autocuidado, alegando que muitas vezes não há com quem deixar a criança ou que o transtorno de pedir para alguém cuidar da criança acarreta em maiores incomodações.

Existe uma adaptação à criança, fazendo que as mães deixem de lado seus papéis ocupacionais, implicando altos níveis de estresse, baixa qualidade de vida e baixo desempenho ocupacional, por ter dificuldade em procurar/ manter emprego, realizar atividades de lazer, interação social com outras pessoas, entre outras coisas.

Todos os membros da família vivenciam a presença do indivíduo deficiente. Porém a mãe é sempre a pessoa que mais agrega sobrecarga emocional e das tarefas do cotidiano. Costuma parar sua vida para viver pelo filho autista, acaba à frente na rotina de cuidados médicos, cuidados pessoais da criança, passando a viver diretamente a criança ${ }^{26}$, deixando de lado seus papéis ocupacionais. Estes são necessários para a satisfação pessoal e a saúde, tornando-se peça-chave para o bem-estar físico e mental ${ }^{32,33,34}$

Segundo os estudos de validação da EPS-10, os níveis de estresse podem variar de 0 a 56 , sendo 56 considerado "estressado ao extremo". Os resultados deste estudo apresentam níveis altos de estresse com média de pontuação 25,26 pontos (Desvio Padrão=7,58); mínimo de 10 e máximo de 38 pontos.

O estudo de validação dessa escala para população brasileira apresentou média geral de estresse de 15,13 (Desvio Padrão=6,459), variando de 5 a 33 pontos, apresentando maiores níveis de estresse na população feminina ${ }^{35}$. Sendo assim, observa-se que as mães cuidadoras de autistas apresentam níveis de estresse superiores à população brasileira.

As diferentes alterações na saúde de uma pessoa levam a mudanças na rotina da família, desencadeando possível estresse e desestabilidade familiar ${ }^{36}$.

Os pais são expostos a uma carga excessiva em razão da rotina de cuidar dos filhos, contudo isso varia conforme a capacidade de cada um em lidar com fatores estressores e com diferentes níveis de tolerância. Na maior parte, esses fatores afetam principalmente as mães, levando ao isolamento social, a um grande estresse, depressão e diminuição da qualidade de vida ${ }^{37 .}$

A atenção em Terapia Ocupacional é fundamental para a mudança de hábitos, para que possa haver a reeducação da rotina, atuando como facilitador da interação das mães com seu contexto/ambiente, atividades e ocupações com o objetivo de alcançar a eficácia apoiando a saúde e a reinserção na vida. É pela reflexão teórica em ações práticas que o Terapeuta Ocupacional atuará em comum acordo com o cliente, promovendo a concordata entre as necessidades objetivas e subjetivas do indivíduo, com o intuito de impactar diretamente na ampliação da saúde global diante da reconstrução/ construção de direitos básicos, como a educação, o lazer, interação social, entre outros, trazendo melhor qualidade de vida, níveis de estresse baixo e desempenho ocupacional adequado ${ }^{17}$.

\section{CONCLUSÕES}

Os dados do estudo sugerem que existe correlação entre as variáveis e que as mães cuidadoras de crianças e adolescentes com autismo apresentam menor qualidade de vida, altos níveis de estresse e desempenho ocupacional baixo.

Acredita-se que, provavelmente, isto ocorre devido à rotina diária das mães, que possuem diversos papéis ocupacionais como mãe, esposa, dona de casa, entre outros, acarretando no acúmulo de tarefas. As características do autismo são os fatores que mais contribuem para esse quadro, visto que as mães administram de diferentes formas as situações em que se apresentam no cotidiano com a criança portadora de autismo.

Sugere-se que novos estudos sejam realizados com essa mesma população com um número amostral maior para confirmar estes resultados. Assim, será possível contribuir para melhor qualidade das evidências científicas e cooperar no esclarecimento das famílias que possuem sujeitos com autismo, a fim de ajudar no desenvolvimento de programas de cuidado e assistência terapêutica para as mães cuidadoras.

Agradecimentos: Ao Centro de Atendimento ao Autista Dr. Danilo Rolim de Moura, pelo auxílio na realização da pesquisa. 


\section{REFERÊNCIAS}

1. Asumpção Jr FB, Sprovieri MH, Kuczynski E, Farinha V. Reconhecimento facial e autismo. Arq Neuro-psiquiatr. 1999;57(4):944-9. DOI: http://dx.doi.org/10.1590/S0004282X1999000600008.

2. Klin A. Autismo e síndrome de Asperger: uma visão geral. Rev Bras Psiquiatr. 2006;28(sup1.1):S3-S11. DOI: http:// dx.doi.org/10.1590/S1516-44462006000500002.

3. American Psychiatric Association. Diagnostic and statistical manual of mental disorders. 5th ed. Arlington, VA: American Psychiatric Publishing; 2013.

4. Elsabbagh M, Divan G, Koh YJ, Kim YS, Kauchali S, Marcín C, Montiel-Nava C, Patel V, Paula CS, Wang C, Yasamy MT, Fombonne E. Global prevalence of autism and other pervasive developmental disorders. Autism Res. 2012;5(3):160-79. DOI: 10.1002/aur239.

5. Zanon RB, Backes B, Bosa CA. Identificação dos primeiros sintomas do autismo pelos pais. Psicol Teor Pesq (Brasília). 2014;30(1):25-33. DOI: http://dx.doi.org/10.1590/S010237722014000100004 .

6. Garcia PM, Mosquera CFF. Causas neurológicas do autismo. Mosaico Rev Pesq Artes Fac Artes Paraná. 2011;(5):106-22. Disponível em: http://periodicos.unespar.edu.br/index.php/ mosaico/article/viewFile/19/pdf.

7. Gupta AR, State MW. Autismo: genética. Rev Bras Psiquiatr. 2006;28(supl I):29-38. Disponível em: http://www.scielo.br/ $\mathrm{pdf} / \mathrm{rbp} / \mathrm{v} 28 \mathrm{~s} 1 / \mathrm{a} 05 \mathrm{v} 28 \mathrm{~s} 1 . \mathrm{pdf}$.

8. Barbaro J, Dissanayake C. Autism spectrum disorders in infancy and toddlerhood: a review of the evidence on early signs, early identification tool, and early diagnosis. J Dev Behav Pediatr. 2009;30(5):447-59. DOI: 10.1097/ DBP.0b013e3181ba0f9f.

9. Costa MIF, Nunesmaia HGS. Diagnóstico genético e clínico do autismo infantil. Arq Neuropsiquiatr. 1998;56(1):24-31. DOI: http://dx.doi.org/10.1590/S0004282X1998000100004.

10. Charman T. Autismo e seu impacto no desenvolvimento infantil. In: Tremblay RE, Barr RG, Peters RDe V, Boivin M, editores. Enciclopédia sobre o desenvolvimento na primeira infância. Montreal: Centre of Excellence for Early; 2011. Disponível em: http://www.enciclopedia-crianca.com/ autismo/segundo-especialistas/autismo-e-seu-impacto-nodesenvolvimento-infantil.

11. Buscaglia L. Os deficientes e seus pais. Um desafio ao aconselhamento. Rio de Janeiro: Record; 1993.

12. Schmidt C, Bosa C. Estresse e auto-eficácia em mães de pessoas com autismo. Arq Bras Psicol (Rio de Janeiro). 2007;59(2):179-91. Disponível em: http://seer.psicologia. ufrj.br/index.php/abp/article/viewFile/88/102.
13. Ornitz EM, Ritvo ER, Gauderer EC. Autismo: revisão crítica da literatura. In: Lipp JRS, organizador. Psiquiatria infantil: estudo multidisciplinar. Belo Horizonte: ABENEDI; 1987. p.113-34.

14. Beck ARM, Lopes MHBM. Tensão devido ao papel de cuidador entre cuidadores de crianças com câncer. Rev Bras Enferm (Brasília). 2007;60(5):513-8. DOI: http://dx.doi. org/10.1590/S0034-71672007000500006

15. Luzardo AR, Gorini MIPC, Silva APSS. Características de idosos com doenças de Alzheimer e seus cuidadores: uma série de casos em um serviço de neurogeriatria. Texto Contexto Enferm (Florianópolis). 2006;15(4):587-94. http:// dx.doi.org/10.1590/S0104-07072006000400006.

16. Lima MG, Barros MBA, César CLG, Goldbaum M, Carandina L, Ciconelli RM. Qualidade de vida relacionada à saúde em idosos, avaliada com o uso do SF-36 em estudo de base populacional. Cad Saúde Pública. 2009;25(10): 2159-67. DOI: http://dx.doi.org/10.1590/S0102-311X2009001000007.

17. Roley SS, DeLany JV, Barrows CJ, Brownrigg S, Honaker D, Sava DI, Talley V, Voelkerding K, Amini DA, Smith E, Toto P, King S, Lieberman D, Baum MC, Cohen ES, Cleveland PA, Youngstrom MJ; American Occupational Therapy Association Commission on Practice. Occupational Therapy Practice. Framework: domain \& process. 2nd. Am J Occup Ther. 2008;63(6):625-83. DOI:10.5014/ajot.62.6.625.

18. Sanini C, Brum EHM, Bosa CA. Depressão materna e implicações sobre o desenvolvimento infantil do autista. Rev Bras Crescimento Desenvolv Hum. 2010;20(3):80915. Disponível em: http://pepsic.bvsalud.org/pdf/rbcdh/ v20n3/16.pdf.

19. Silva NLP, Dessen MA. O que significa ter uma criança com deficiência mental na família? Educar (Curitiba). 2004;23:161-83. Disponível em: http://ojs.c3sl.ufpr.br/ojs/ index.php/educar/article/view/2158/1810.

20. Law M. Medida Canadense de Desempenho Ocupacional (COPM). Trad. Org. Cardoso AA, Magalhães LC. Belo Horizonte: Ed. UFMG; 2009.

21. Fleck MPA Louzada S, Xavier M, Chachamovich E, Vieira G, Santos L, Pinzon V. Aplicação da versão em português do instrumento abreviado de avaliação da qualidade de vida "WHOQOL-bref". Rev Saúde Pública. 2000;34(2):178-83. DOI: http://dx.doi.org/10.1590/S0034-89102000000200012.

22. Homann D, Stefanello JMF, Góes SM, Breda CA, Paiva ES, Leite N. Percepção de estresse e sintomas depressivos: funcionalidade e impacto na qualidade de vida em mulheres com fibromialgia. Rev Bras Reumatol. 2012;52(3):324-30. DOI: http://dx.doi.org/10.1590/S0482-50042012000300003.

23. Hulley SB, et al. Delineando a pesquisa clínica: uma abordagem epidemiológica. Porto Alegre: Artmed; 2008. 
24. Silva ABB, Gaiato MB, Reveles LT. Mundo singular entenda o autismo. Rio de Janeiro: Fontanar; 2012.

25. Correia EMAM. Proficiência motora em crianças e jovens com síndrome de Asperger [Dissertação]. Lisboa: Faculdade de Motricidade Humana; 2012. Disponível em: http://hdl. handle.net/10400.5/4469.

26. Barbirato F, Dias G. A mente do seu filho: como estimular as crianças e identificar os distúrbios psicológicos na infância. Rio de Janeiro: AGIR; 2009.

27. Fávero BAM, Santos AM. Autismo infantil e estresse familiar: uma revisão sistemática da literatura. Psicol Reflexão Crítica (Porto Alegre). 2005;18(3):358-69. Disponível em: http:// www.scielo.br/pdf/prc/v18n3/a10v18n3.

28. Almeida Pimenta R, Rodrigues LA, Greguol M. Avaliação da qualidade de vida e sobrecarga de cuidadores de pessoas com deficiência intelectual. Rev Bras Cien Saúde. 2010;14(3): 69-76. DOI: 10.4034/RBCS.2010.14.03.10

29. Cuvero MM. Qualidade de vida relacionada em cuidadores de crianças e adolescentes com autismo [Dissertação]. Uberlândia: Universidade Federal de Uberlândia; 2008. Disponível em: http://hdl.handle.net/123456789/2736.

30. Nóbrega KID, Pereira CU. Qualidade de vida, ansiedade e depressão em cuidadores de crianças com neoplasia cerebral. Psicol Teor Prát. 2001;13(1):48-61. Disponível em: http:// pepsic.bvsalud.org/pdf/ptp/v13n1/v13n1a04.pdf.
31. Garrido RA, Osvaldo P. Distúrbios de comportamento em pacientes com demência: impacto sobre a vida do cuidador. Arq Neuropsiquiatr. 1999;57(2B):427-34. DOI: http://dx.doi. org/10.1590/S0004-282X1999000300014.

32. Serra D. Autismo, família e inclusão. Polêm!ca. 2010;9(1):4056. Disponível em: http://www.e-publicacoes.uerj.br/index. php/polemica/article/view/2693/1854.

33. Hagedorn R. Ferramentas para a prática em terapia ocupacional. São Paulo: Editora Roca; 2007.

34. Marcon SS, Sassá AH, Soares NTI, Molina RCM. Dificuldade e conflitos enfrentados pela família no cuidado cotidiano a uma criança com doença crônica. Cienc Cuid Saúde. 2007;6(Supl 2):411-9.

35. Luft CDB, Sanches SO, Mazo GZ, Andrade A. Versão brasileira da escala de estresse percebido: tradução e validação para idosos. Rev Saúde Pública. 2007;41(4):606-15. DOI: http://dx.doi.org/10.1590/S0034-89102007000400015.

36. Luzardo AR, Waldman BF. Atenção ao familiar cuidador do idoso com doença de Alzheimer. Acta Sci Health Sci (Maringá). 2004;26(1):135-45. Disponível em: http:// periodicos.uem.br/ojs/index.php/ActaSciHealthSci/article/ viewFile/1640/1067.

37. Miura RT, Petean EBL. Paralisia cerebral grave: o impacto na qualidade de vida de mães cuidadoras. Mudanças Psicol Saúde. 2012;20(1-2):7-12. DOI: http://dx.doi. org/10.15603/2176-1019/mud.v20n1-2p7-12. 